\title{
Effect of density of fingerling and juvenile pirarucu during transportation on water quality and physiological parameters
}

\author{
Adriana Ferreira LIMA ${ }^{1 *}$, , Hyago Jovane Borges de OLIVEIRA², André Silvério PEREIRA ${ }^{3}$, \\ Silmara Sanae SAKAMOTO3 \\ Empresa Brasileira de Pesquisa Agropecuária - Embrapa Pesca e Aquicultura, NS 10, cruzamento com a LO 18, Loteamento Água Fria, Palmas, Tocantins, Brazil \\ 2 Universidade Federal de Santa Catarina, Centro de Ciências Agrárias, Departamento de Aquicultura, Programa de Pós Graduação em Aquicultura, Rodovia Admar \\ Gonzaga 1346, Florianópolis, SC, Brazil \\ 3 Faculdade Católica do Tocantins - FACTO, Rodovia TO-050, Loteamento Coqueirinho, Lote 7, Palmas, TO, Brazil \\ * Corresponding author: adriana.lima@embrapa.br; 으 https://orcid.org/0000-0002-8828-6992
}

\section{ABSTRACT}

We assessed the effect of stocking density on physiological parameters (blood lactate, glucose, cortisol, hematocrit), water quality (temperature, dissolved oxygen, $\mathrm{pH}$, unionized ammonia, carbon dioxide), and survival during the transportation of fingerling $(24.5 \pm 4.7 \mathrm{~g})$ and juvenile $(615.8 \pm 122.2 \mathrm{~g})$ pirarucu (Arapaima gigas) for six hours in plastic bags. The tested densities were 65, 80, 95, 110 and $125 \mathrm{~g} \mathrm{~L}^{-1}$ for fingerlings, and 50, 80,110, 140 and $170 \mathrm{~g} \mathrm{~L}^{-1}$ for juveniles (three replicates each). Parameters were measured prior to and immediately after transportation, and at 24 and 96 hours recovery after transportation. No mortality was observed, except for fingerlings $(<3 \%)$ at densities of 110 and $125 \mathrm{~g} \mathrm{~L}^{-1}$ during recovery. All the water quality parameters were significantly altered after the transportation of fingerlings and juveniles. Water temperature, dissolved oxygen, carbon dioxide and unionized ammonia increased, but $\mathrm{pH}$ decreased. Only carbon dioxide and unionized ammonia differed among densities. Cortisol levels did not increase over time, except for the juveniles at $170 \mathrm{~g} \mathrm{~L}^{-1}$, which still had high cortisol after 96 hours. Glucose significantly increased after transportation for all the treatments and returned to the initial values during the recovery period. Conversely, the lactate values were still high after 96 hours. Hematocrit was assessed only for juveniles and was significantly lower after transportation. We conclude that fingerling and juvenile pirarucu can be safely transported at densities up to $95 \mathrm{~g} \mathrm{~L}^{-1}$ and $140 \mathrm{~g} \mathrm{~L}^{-1}$, respectively.

KEYWORDS: Arapaima gigas, closed system, lactate, cortisol, glucose

\section{Efeito da densidade de transporte de alevinos e juvenis de pirarucu sobre a qualidade da água e parâmetros fisiológicos}

\section{RESUMO}

Este estudo avaliou o efeito da densidade de estocagem sobre parâmetros fisiológicos (lactato, glicose, cortisol e hematócrito), qualidade de água (temperatura, oxigênio dissolvido, $\mathrm{pH}$, amônia não ionizada e gás carbônico) e sobrevivência no transporte de alevinos $(24.5 \pm 4.7 \mathrm{~g})$ e juvenis $(615.83 \pm 122.19 \mathrm{~g})$ de pirarucu (Arapaima gigas) em sacos plásticos por seis horas. Avaliamos densidades de 65, 80, 95, 110 e $125 \mathrm{~g} \mathrm{~L}^{-1}$ para alevinos, e de 50, 80, 110, 140 e $170 \mathrm{~g} \mathrm{~L}^{-1}$ para juvenis (três réplicas cada). Os parâmetros de resposta foram medidos antes e logo após o transporte, e após 24 e 96 horas de recuperação. Não foi observada mortalidade, exceto para alevinos (<3\%) das densidades de 110 e $125 \mathrm{~g} \mathrm{~L}^{-1}$ durante o período de recuperação. Foram observadas alteraçóes significativas em todos os parâmetros de qualidade de água após o transporte de alevinos e juvenis. Houve elevação da temperatura, oxigênio dissolvido, gás carbônico e amônia não ionizada e decréscimo do $\mathrm{pH}$, com diferenças entre as densidades apenas para gás carbônico e amônia não ionizada. Não houve elevaçáo dos níveis de cortisol, exceto para juvenis na densidade de $170 \mathrm{~g} \mathrm{~L}^{-1}$ que ainda permaneciam com nível alto de cortisol às 96 horas. A glicose se elevou após o transporte em todos os tratamentos, retornando aos valores iniciais na recuperação, ao contrário do lactato, que se elevou e permaneceu alto até as 96 horas. O hematócrito foi avaliado apenas para juvenis e diminuiu significativamente após o transporte. Concluímos que alevinos de pirarucu podem ser transportados com segurança em densidades de até $95 \mathrm{~g}$ $\mathrm{L}^{-1} \mathrm{e}$ juvenis em densidades de até $140 \mathrm{~g} \mathrm{~L}^{-1}$.

PALAVRAS-CHAVE: Arapaima gigas, sistema fechado, lactato, cortisol, glicose 


\section{INTRODUCTION}

Live fish transportation is a routine practice in fish farming that allows fish to be moved between production chain links. During transportation, fish are simultaneously subject to several stressors, mainly long exposure to poor water quality and inappropriate stocking densities, and to poor handling, confinement and acclimation (Harmon 2009). These stressors, in conjunction with fish health status and the level of blood chemical changes, are the main factors to affect post-transportation survival (Berka 1986; Portz et al. 2006).

Fish may be transported in closed systems and all conditions for survival must be provided while fish are confined (Berka 1986). As closed systems offer no air or water exchanges, loading capacity is an important determinant for fish safety during transportation. Water quality is one of the main factors to determine loading capacity during transportation (Portz et al. 2006) and is a limiting factor of stocking density during transportation as water deterioration increases with fish density because metabolic residue accumulates (Lim et al. 2003). High densities may also cause injuries to fish (King 2009; Ross and Ross 2008). Moreover, there is pressure from the production sector for higher stocking densities to improve economic viability (Portz et al. 2006; Carneiro et al. 2009).

With increasing pirarucu, Arapaima gigas (Schinz 1822) farming in Brazil (IBGE 2018), transporting fish of different size classes to connect production sector links has intensified, mainly between hatcheries and grow-out farms, which frequently demand fish as long as $20 \mathrm{~cm}$. Fish larger than $20 \mathrm{~cm}$ are generally demanded by producers whose production structures are deficient (Rebelatto et al. 2015). Nevertheless, very few studies have assessed transportation variables. Brandão et al. (2006) assessed the physiological effects of transporting pirarucu $(33 \mathrm{~g})$ in plastic bags at a density of $20 \mathrm{~g} \mathrm{~L}^{-1}$ for three hours, and reported a rise in glucose levels and lower lactate levels after transportation, but no mortality. Gomes et al. (2006) reported no fish mortality after transporting pirarucu ( $33 \mathrm{~g})$ in plastic bags at a density of $40 \mathrm{~g} \mathrm{~L}^{-1}$ for three hours at different $\mathrm{NaCl}$ concentrations $\left(0,1,3,5 \mathrm{~g} \mathrm{~L}^{-1}\right)$, but $\mathrm{Na}^{+}, \mathrm{Cl}^{-}$and $\mathrm{Ca}^{2+}$ net fluxes increased and salt levels were not recommended. Gomes et al. (2003) assessed the transportation of pirarucu (1.1 kg, 1 fish per bag) in plastic bags $(10 \mathrm{~L}$ water) for six hours with and without oxygen supply. They reported no mortality, although glucose levels were higher, which indicates stress. Lima and Oliveira (2018) evaluated three densities $\left(80,120,160 \mathrm{~kg} \mathrm{~m}^{-3}\right)$ for the transportation of pirarucu $(9 \mathrm{~kg})$ in an open system. They did not reach the maximum loading capacity, noted no mortality. However, hematocrit values were higher, lactate concentrations were lower, and they observed alterations in water quality parameters (higher ammonia and carbon dioxide $\left(\mathrm{CO}_{2}\right)$ concentrations, and less dissolved oxygen).
The gap in information on adequate pirarucu transportation still exists, mainly concerning basic aspects such as the effect of density and how it directly affects costs. Therefore, the present study aimed to assess the effect of different stocking densities on water quality, physiological stress parameters and mortality while transporting fingerlings and juveniles pirarucu in plastic bags for six hours.

\section{MATERIAL AND METHODS}

\section{Experimental design}

The present study was conducted by performing two transport trials for fingerling and juvenile pirarucu, respectively. Prior to transportation, fish from both size classes were kept in $300 \mathrm{~L}$ tanks $\left(1-2 \mathrm{~g} \mathrm{~L}^{-1}\right)$ and fed ad libitum a commercial extruded feed for carnivorous fish species $(40 \%$ crude protein) three times a day. The fish transportation route included paved roads and back roads, and lasted 6 hours (h), considered a short transportation time (Sampaio and Freire 2016). This time allows the producer to sell fish in cities at a distance of up to some $400 \mathrm{~km}$ away, which allows providing a service to at least an entire state in north Brazil.

Fingerlings had mean \pm SD for weight and length of $24.5 \pm 4.7 \mathrm{~g}$ and $15.6 \pm 1.0 \mathrm{~cm}$, respectively. The trial was conducted according to a completely randomized design with five stocking densities $\left(65,80,95,110,125 \mathrm{~g} \mathrm{~L}^{-1}\right)$ and three replicates, which totaled 15 experimental units. Fish were transported in 60-liter plastic bags containing $5 \mathrm{~L}$ of water, completely filled with pure oxygen after fish were stocked in bags.

Juveniles had mean \pm SD weight and length of 615.8 $\pm 122.2 \mathrm{~g}$ and $47.5 \pm 6.3 \mathrm{~cm}$, respectively. The trial was conducted according to a completely randomized design with five stocking densities $\left(50,80,110,140,170 \mathrm{gL}^{-1}\right)$ and three replicates, which gave 15 experimental units. Fish were transported in 60 -liter plastic bags containing $10 \mathrm{~L}$ of water, completely filled with pure oxygen after fish were stocked in bags.

To minimize the influence of handling stress on transportation, two days before the trials began, fish were captured and immobilized for biometric measurements (weight and total length) (Cupp et al. 2017). Then they were distributed in the $300 \mathrm{~L}$ tanks in the densities informed above at a constant water flow $\left(4.5 \mathrm{~L} \mathrm{~min}^{-1}\right)$ until they were packed for shipping. On the day of transportation, fish were captured from tanks with a dip net, blood was sampled from the caudal vein and fish were transferred to plastic bags. After transportation, fish were placed in 15 separate recovery tanks $(300 \mathrm{~L})$. Feeding was suspended for $24 \mathrm{~h}$ before and after transportation to empty the fish digestive tract. Then fish were fed for the following $72 \mathrm{~h}$, when they were fasted again for blood sampling $96 \mathrm{~h}$ after transportation. Accumulated 
mortality was recorded immediately after transportation and during the 96-hour recovery period.

For the physiological parameters in the fingerling trial, blood from two fish per experimental unit (six per treatment) was sampled before, immediately after, $24 \mathrm{~h}$ and $96 \mathrm{~h}$ after transportation. Blood samples were collected from the caudal vasculature with EDTA-treated syringes in less than 1 minute without anesthesia. The fish used for blood sampling were not returned to the experimental units. Due to the densitydetermined lower number of juveniles per treatment, blood was sampled from all the fish for the physiological parameters and fish were returned to the experimental units.

\section{Water analysis}

Water quality parameters were measured immediately before and after transportation. As the same water source was used to fill the bags in each experiment, only one sample of source water was analyzed, as a control for water condition immediately before transportation. After transportation, one water sample was taken per experimental unit (three per treatment). Water $\mathrm{pH}$, dissolved oxygen, unionized ammonia and temperature were measured by a probe (YSI, Yellow Springs, USA). Carbon dioxide was measured with a commercial colorimetric kit (Alfakit, Florianópolis, SC, Brazil).

\section{Physiological analysis}

Fish physiological parameters were assessed by measuring the following blood indicators: cortisol ( $\left.\mathrm{ng} \mathrm{dL}^{-1}\right)$, glucose ( $\mathrm{mg}$ $\left.\mathrm{dL}^{-1}\right)$ and lactate $\left(\mathrm{mg} \mathrm{dL}^{-1}\right)$ for both trials and hematocrit (\%) only in juveniles due to insufficient blood volume sampled in fingerlings. Hematocrit was determined after centrifuging blood (1387 g, $5 \mathrm{~min}$ ) in microcapillary tubes. The microplate enzyme immune assay (Monobind, Lake Forest, CA, USA) was used to determine the blood cortisol concentration. Blood glucose and lactate were determined by the enzymatic method (Labtest, Lagoa Santa, MG, Brazil). The analyses were performed at Empresa Brasileira de Pesquisa Agropecuária (Palmas, Tocantins, Brazil).

\section{Statistical analysis}

The same statistical analyses were performed for the fingerling and juvenile data. Mortality and water quality parameters after transport were compared by a one-way ANOVA, followed by a Tukey test $(\mathrm{p}<0.05)$. Differences between the control and water quality parameters after transportation were compared by a Student's t-test ( $\mathrm{p}<0.05)$. The physiological stress indicators were compared among treatments by a two-way ANOVA, with density and transport period as factors, followed by a Tukey test $(\mathrm{p}<0.05)$. To evaluate the homogeneity of variance and normality premises, the Bartlett and the Shapiro-Wilk tests were respectively performed. When premises were not met, data were BoxCox-transformed prior to the analysis. Statistical analyses were performed in R (R Core Team 2016).

\section{Ethics and legal aspects}

The study complied with Brazilian guidelines for the care and use of animals for scientific and educational purposes being approved by the Ethics Committee for the Use of Animals-CEUA of the National Research Center on Fisheries, Aquaculture and Agricultural Systems-CNPASA (protocol nos. 34/2017 and 40/2018).

\section{RESULTS}

\section{Fingerling trial}

No mortality was observed immediately after transportation. However by the end of the recovery period, mortality was recorded at the higher densities of 110 and 125 $\mathrm{g} \mathrm{L}^{-1}$ (Table 1). Temperature, dissolved oxygen, unionized ammonia and $\mathrm{CO}_{2}$ increased after transportation, but $\mathrm{pH}$ decreased. Water $\mathrm{pH}$, temperature, dissolved oxygen and $\mathrm{CO}_{2}$ concentration did not vary significantly among densities

Table 1. Overall mortality ( $96 \mathrm{~h}$ after transportation) of fingerlings of pirarucu, Arapaima gigas and water quality parameters immediately before (control) and after fingerling transportation for six hours in plastic bags at densities of $65,80,95,110$ and $125 \mathrm{~g} \mathrm{~L}^{-1}$. Values are the mean \pm standard deviation of three replicates.

\begin{tabular}{|c|c|c|c|c|c|c|}
\hline \multirow{2}{*}{ Water parameter } & \multirow{2}{*}{ Control } & \multicolumn{5}{|c|}{ After transportation } \\
\hline & & $65 \mathrm{gL}^{-1}$ & $80 \mathrm{gL}^{-1}$ & $95 \mathrm{gL}^{-1}$ & $110 \mathrm{gL}^{-1}$ & $125 \mathrm{gL}^{-1}$ \\
\hline Temperature $\left({ }^{\circ} \mathrm{C}\right)$ & $27.1 \mathrm{~A}$ & $31.87 \pm 0.46 \mathrm{Ba}$ & $31.63 \pm 0.85 \mathrm{Ba}$ & $31.23 \pm 0.25 \mathrm{Ba}$ & $31,27 \pm 0.35 \mathrm{Ba}$ & $33.90 \pm 4.31 \mathrm{Aa}$ \\
\hline Dissolved oxygen $\left(\mathrm{mgL}^{-1}\right)$ & $2.37 \mathrm{~A}$ & $6.85 \pm 0.55 \mathrm{Ba}$ & $6.05 \pm 0.68 \mathrm{Ba}$ & $6.53 \pm 0.21 \mathrm{Ba}$ & $6.59 \pm 0.42 \mathrm{Ba}$ & $6.26 \pm 0.59 \mathrm{Aa}$ \\
\hline $\mathrm{pH}$ & $7.31 \mathrm{~A}$ & $6.62 \pm 0.13 \mathrm{Ba}$ & $6.51 \pm 0.13 \mathrm{Ba}$ & $6.43 \pm 0.04 \mathrm{Ba}$ & $6.42 \pm 0.01 \mathrm{Ba}$ & $6.42 \pm 0.11 \mathrm{Ba}$ \\
\hline Unionized ammonia (mgL-1) & $0.05 \mathrm{~A}$ & $1.62 \pm 0.26 \mathrm{Bd}$ & $1.94 \pm 0.11 \mathrm{Bd}$ & $2.39 \pm 0.12 \mathrm{BC}$ & $3.08 \pm 0.02 \mathrm{Bb}$ & $3.67 \pm 0.17 \mathrm{Ba}$ \\
\hline Carbon dioxide $\left(\mathrm{mgL}^{-1}\right)$ & $2.0 \mathrm{~A}$ & $12.67 \pm 6.43 \mathrm{Aa}$ & $10.67 \pm 1.15 \mathrm{Ba}$ & $10.33 \pm 4.04 \mathrm{Aa}$ & $14.33 \pm 6.03 \mathrm{Aa}$ & $13.67 \pm 5.13 \mathrm{Aa}$ \\
\hline Overall mortality (\%) & - & $0.00 \mathrm{~b}$ & $0.00 \mathrm{~b}$ & $0.00 \mathrm{~b}$ & $2.67 \pm 3.06 a$ & $0.33 \pm 0.58 \mathrm{ab}$ \\
\hline
\end{tabular}

Different lowercase letters indicate significant differences among treatments (one-way ANOVA, followed by a Tukey test at $p<0.05$ ). Uppercase letters denote significant differences between the control and values after transportation (Student's t-test at $\mathrm{p}<0.05$ ). 
immediately after transportation, but unionized ammonia rose with increased density (Table 1).

The blood glucose concentration was influenced significantly by density and time (immediately before, after, and at $24 \mathrm{~h}$ and $96 \mathrm{~h}$ after transportation). Higher concentrations were observed immediately after transportation, which returned to the initial values by the end of the recovery period for all the tested densities (Figure 1; Table 2).

The blood lactate concentration did not differ significantly between densities. With time however, i.e., significantly higher values were observed immediately after transportation that decreased during the recovery period, but did not return to the initial values. The blood cortisol levels did not increase with either time or density.

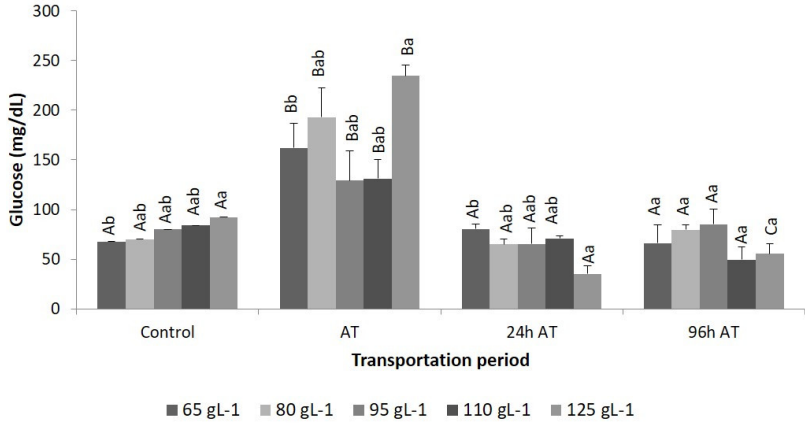

Figure 1. Mean plasma glucose concentration $\left(\mathrm{mg} \mathrm{dL}^{-1}\right)$ during the transportation procedure of pirarucu, Arapaima gigas fingerlings at different densities. Control before transport; AT - immediately after transport, $24 \mathrm{~h}$ AT - $24 \mathrm{~h}$ after transport; $96 \mathrm{~h}$ AT $-96 \mathrm{~h}$ after transport. Different lowercase superscript letters indicate significant differences between densities. Uppercase letters denote differences between transportation periods (Tukey, $\mathrm{p}<0.05$ )

Table 2. Cortisol, lactate and glucose levels in pirarucu Arapaima gigas fingerlings at different densities and at different times during the transport process. Control before transport; AT - immediately after transport, $24 \mathrm{~h} \mathrm{AT} \mathrm{-} 24$ hours after transport; $96 \mathrm{~h}$ AT - 96 hours after transport. Values are the mean \pm standard deviation of three replicates. The significance of the two-way ANOVA for each dependent variable is also shown.

\begin{tabular}{|c|c|c|c|c|}
\hline $\begin{array}{l}\text { Density } \\
\left(\mathrm{g} \mathrm{L}^{-1}\right)\end{array}$ & Transportation period & $\begin{array}{l}\text { Cortisol } \\
\left(\mu \mathrm{gdL}^{-1}\right)\end{array}$ & $\begin{array}{l}\text { Lactate } \\
\left(\mathrm{mg} \mathrm{dL}^{-1}\right)\end{array}$ & $\begin{array}{l}\text { Glucose } \\
\left(\mathrm{mg} \mathrm{dL}^{-1}\right)\end{array}$ \\
\hline \multirow[t]{4}{*}{65} & Control & $4.89 \pm 4.58$ & $9.74 \pm 0.73 \mathrm{Ca}$ & $67.54 \pm 11.69 \mathrm{Ab}$ \\
\hline & AT & $7.55 \pm 5.26$ & $24.44 \pm 2.08 \mathrm{Aa}$ & $161.90 \pm 24.71 \mathrm{Bb}$ \\
\hline & $24 \mathrm{~h} \mathrm{AT}$ & $2.73 \pm 0.75$ & $25.81 \pm 10.56 \mathrm{Aa}$ & $80.31 \pm 4.95 \mathrm{Ab}$ \\
\hline & $96 \mathrm{~h} \mathrm{AT}$ & $4.86 \pm 4.17$ & $20.17 \pm 10.18 \mathrm{Ba}$ & $65.99 \pm 18.75 \mathrm{Aa}$ \\
\hline \multirow[t]{4}{*}{80} & Control & $6.55 \pm 0.27$ & $8.21 \pm 1.45 \mathrm{Ca}$ & $69.96 \pm 26.52 \mathrm{Aab}$ \\
\hline & AT & $8.75 \pm 6.28$ & $25.98 \pm 3.41 \mathrm{Aa}$ & $193.15 \pm 29.37 \mathrm{Bab}$ \\
\hline & $24 \mathrm{~h}$ AT & $8.12 \pm 0.81$ & $27.35 \pm 8.34 \mathrm{Aa}$ & $65.12 \pm 5.54 \mathrm{Aab}$ \\
\hline & $96 \mathrm{~h} \mathrm{AT}$ & $5.69 \pm 3.79$ & $14.02 \pm 10.87 \mathrm{Ba}$ & $79.70 \pm 4.76 \mathrm{Aa}$ \\
\hline \multirow[t]{4}{*}{95} & Control & $7.71 \pm 2.60$ & $9.23 \pm 2.90 \mathrm{Ca}$ & $80.04 \pm 6.56 \mathrm{Aab}$ \\
\hline & AT & $11.04 \pm 8.25$ & $30.26 \pm 4.89 \mathrm{Aa}$ & $129.03 \pm 30.22 \mathrm{Bab}$ \\
\hline & $24 \mathrm{~h} \mathrm{AT}$ & $7.95 \pm 2.52$ & $22.05 \pm 3.12 \mathrm{Aa}$ & $65.39 \pm 16.21$ Aab \\
\hline & $96 \mathrm{~h} \mathrm{AT}$ & $7.34 \pm 3.27$ & $19.32 \pm 3.49 \mathrm{Ba}$ & $85.28 \pm 15.14 \mathrm{Aa}$ \\
\hline \multirow[t]{4}{*}{110} & Control & $4.92 \pm 6.93$ & $6.67 \pm 5.08 \mathrm{Ca}$ & $83.87 \pm 11.40$ Aab \\
\hline & AT & $11.92 \pm 10.22$ & $29.57 \pm 0.78 \mathrm{Aa}$ & $131.12 \pm 19.25 \mathrm{Bab}$ \\
\hline & $24 \mathrm{~h} \mathrm{AT}$ & $13.08 \pm 8.05$ & $23.25 \pm 9.82 \mathrm{Aa}$ & $70.97 \pm 2.71 \mathrm{Aab}$ \\
\hline & $96 \mathrm{~h} \mathrm{AT}$ & $4.58 \pm 0.15$ & $16.58 \pm 6.92 \mathrm{Ba}$ & $49.40 \pm 12.69 \mathrm{Aa}$ \\
\hline \multirow[t]{4}{*}{125} & Control & $5.50 \pm 2.87$ & $5.64 \pm 3.63 \mathrm{Ca}$ & $92.14 \pm 8.27 \mathrm{Aa}$ \\
\hline & AT & $12.05 \pm 5.98$ & $18.29 \pm 8.34 \mathrm{Aa}$ & $234.74 \pm 11.13 \mathrm{Ba}$ \\
\hline & $24 \mathrm{~h} \mathrm{AT}$ & $2.13 \pm 1.07$ & $10.43 \pm 0.36 \mathrm{Aa}$ & $35.08 \pm 7.98 \mathrm{Aa}$ \\
\hline & $96 \mathrm{~h} \mathrm{AT}$ & $6.87 \pm 4.96$ & $13.16 \pm 9.21 \mathrm{Ba}$ & $55.38 \pm 9.98 \mathrm{Ca}$ \\
\hline \multicolumn{2}{|c|}{ Density (D) } & ns & ns & $p<0.01$ \\
\hline \multicolumn{2}{|c|}{ Transportation period (P) } & ns & $p<0.001$ & $p<0.001$ \\
\hline \multicolumn{2}{|c|}{ DxP interaction } & ns & ns & $p<0.01$ \\
\hline
\end{tabular}

Different lowercase letters indicate significant differences between densities and uppercase letters denote differences between transportation periods (Tukey test, $\mathrm{P}$ $<0.05)$; ns (not significant). 


\section{Juvenile trial}

No mortality was observed immediately after transportation or during the 96-hour recovery period. Temperature, dissolved oxygen, unionized ammonia and $\mathrm{CO}_{2}$ increased significantly after transportation, but $\mathrm{pH}$ decreased. Water dissolved oxygen concentration, $\mathrm{pH}$ and temperature did not differ significantly among densities immediately after transportation, whereas unionized ammonia and $\mathrm{CO}_{2}$ increased with density (Table 3).

Juvenile pirarucu physiological responses were significantly influenced by the density/time interaction (immediately before, after, and $24 \mathrm{~h}$ and $96 \mathrm{~h}$ after transportation), except the blood lactate levels (Table 4).

Blood lactate was higher after transportation at higher densities and did not return to the initial levels after the recovery period. Cortisol levels did not increase significantly with transportation, except for the $170 \mathrm{~g} \mathrm{~L}^{-1}$ density, which increased after transportation and did not return to the initial values after the recovery period (Figure 2). Glucose levels

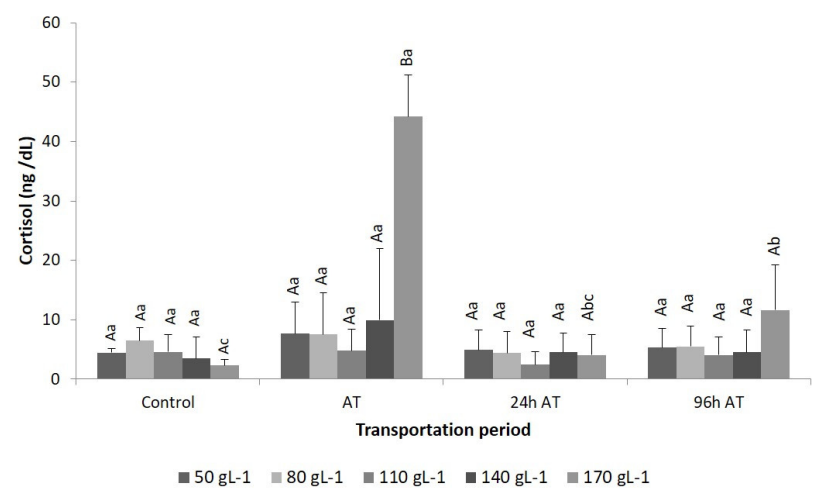

Figure 2. Mean plasma cortisol concentration (ng dL ${ }^{-1}$ ) during the transportation procedure of pirarucu, Arapaima gigas juveniles at different densities. Control before transport; AT - immediately after transport, $24 \mathrm{~h} \mathrm{AT}-24 \mathrm{~h}$ after transport; $96 \mathrm{~h}$ AT - $96 \mathrm{~h}$ after transport. Different uppercase superscript letters indicate significant differences between densities. Lowercase letters denote difference between transportation periods (Tukey, $\mathrm{p}<0.05$ ). increased after transportation for most densities and returned to the initial values during the recovery period $(24 \mathrm{~h}$ and 96 h) (Figure 3a). Hematocrit levels decreased significantly after transportation and during the recovery period for all the densities, and were lowest for the 140 and $170 \mathrm{~g} \mathrm{~L}^{-1}$ densities (Figure 3b).

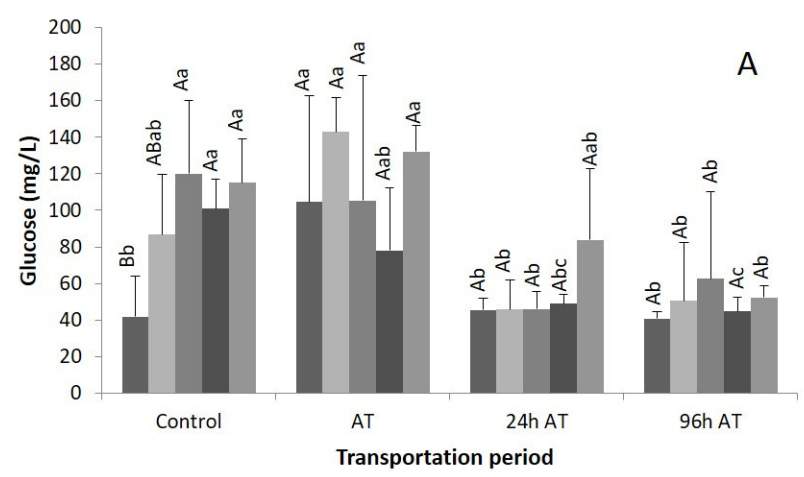

च $50 \mathrm{gL}-1 \quad \square 0 \mathrm{gL}-1 \quad \square 110 \mathrm{gL}-1 \quad \square 140 \mathrm{gL}-1 \quad \square 170 \mathrm{gL}-1$

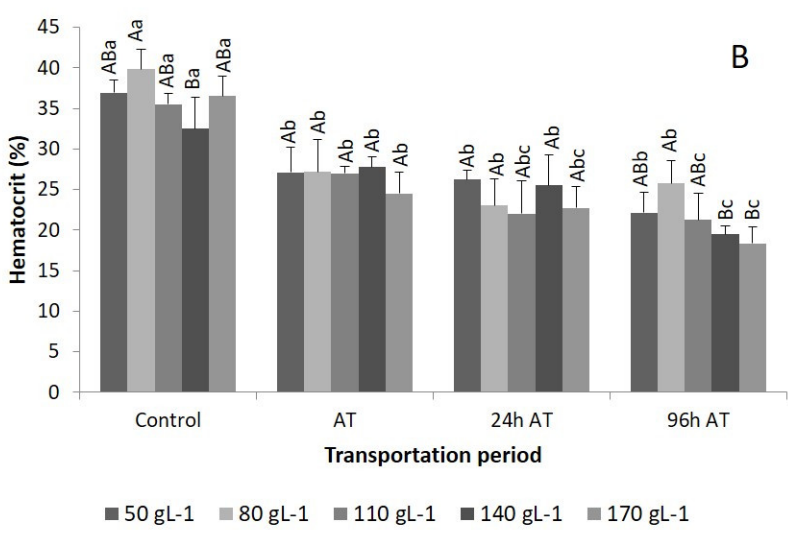

Figure 3. Mean plasma glucose concentrations ( $\left.\mathrm{mg} \mathrm{dL}^{-1}\right)(\mathrm{A})$ and hematocrit (\%) (B) during the transportation procedure of pirarucu, Arapaima gigas juveniles at different densities. Control - before transport; AT - immediately after transport, $24 \mathrm{~h}$ AT - $24 \mathrm{~h}$ after transport; $96 \mathrm{~h}$ AT - $96 \mathrm{~h}$ after transport. Different uppercase superscript letters indicate significant differences between densities. Lowercase letters denote differences between transportation periods (Tukey, $\mathrm{p}<0.05$ ).

Table 3. Water quality parameters immediately before (control) and after juvenile of pirarucu Arapaima gigas transportation for six hours in plastic bags at densities of $65,80,95,110$ and $125 \mathrm{~g} \mathrm{~L}^{-1}$. Values are the mean \pm standard deviation of three replicates.

\begin{tabular}{|c|c|c|c|c|c|c|}
\hline \multirow{2}{*}{ Water parameter } & \multirow{2}{*}{ Control } & \multicolumn{5}{|c|}{ After transportation } \\
\hline & & $50 \mathrm{~g} \mathrm{~L}^{-1}$ & $80 \mathrm{~g} \mathrm{~L}^{-1}$ & $110 \mathrm{~g} \mathrm{~L}^{-1}$ & $140 \mathrm{~g} \mathrm{~L}^{-1}$ & $170 \mathrm{~g} \mathrm{~L}^{-1}$ \\
\hline Temperature $\left({ }^{\circ} \mathrm{C}\right)$ & $27.8 \mathrm{~A}$ & $31.75 \pm 0.15 \mathrm{Ba}$ & $31.97 \pm 0.17 \mathrm{Ba}$ & $31.55 \pm 0.13 \mathrm{Ba}$ & $31.4 \pm 0.08 \mathrm{Ba}$ & $31.42 \pm 0.05 \mathrm{Ba}$ \\
\hline Dissolved oxygen (mg L $\mathrm{L}^{-1}$ ) & $5.55 \mathrm{~A}$ & $16.92 \pm 1.93 \mathrm{Ba}$ & $15.7 \pm 0.74 \mathrm{Ba}$ & $16.23 \pm 2.89 \mathrm{Ba}$ & $14.91 \pm 2.48 \mathrm{Ba}$ & $15.24 \pm 1.98 \mathrm{Ba}$ \\
\hline pH & $6.79 \mathrm{~A}$ & $6.26 \pm 0.10 \mathrm{Ba}$ & $6.17 \pm 0.10 \mathrm{Ba}$ & $6.12 \pm 0.07 \mathrm{Ba}$ & $6.15 \pm 0.05 \mathrm{Ba}$ & $6.16 \pm 0.07 \mathrm{Ba}$ \\
\hline Unionized ammonia ( $\mathrm{mg} \mathrm{L}^{-1}$ ) & $0.05 \mathrm{~A}$ & $0.32 \pm 0.09 \mathrm{BC}$ & $0.50 \pm 0.10 \mathrm{BC}$ & $0.80 \pm 0.10 \mathrm{Bb}$ & $0.94 \pm 0.07 \mathrm{Bb}$ & $1.34 \pm 0.17 \mathrm{Ba}$ \\
\hline Carbon dioxide $\left(\mathrm{mg} \mathrm{L}^{-1}\right)$ & $2.0 \mathrm{~A}$ & $3.88 \pm 0.63 \mathrm{BC}$ & $4.08 \pm 0.00 \mathrm{BC}$ & $8.25 \pm 1.94 \mathrm{Bb}$ & $7.87 \pm 1.44 \mathrm{Bb}$ & $11.7 \pm 0.48 \mathrm{Ba}$ \\
\hline
\end{tabular}

Different lowercase letters indicate significant differences among treatments (one-way ANOVA, followed by Tukey test $p<0.05$ ). Uppercase letters denote significant differences between the control and after the transportation values, Student's t-test $(p<0.05)$. 
Table 4. Cortisol, lactate, glucose and hematocrit levels in pirarucu Arapaima gigas juvenile at different densities and for distinct transport process periods. Control - before transport; AT - immediately after transport, $24 \mathrm{~h}$ AT - $24 \mathrm{~h}$ after transport; $96 \mathrm{~h} \mathrm{AT} \mathrm{-} 96 \mathrm{~h}$ after transport. Values are the mean \pm standard deviation of three replicates. The significance of the two-way ANOVA for each dependent variable is also shown.

\begin{tabular}{|c|c|c|c|c|c|}
\hline Density $\left(g L^{-1}\right)$ & Transportation period & Cortisol $\left(\mu \mathrm{g} \mathrm{dL^{-1 } )}\right.$ & Lactate $\left(\mathrm{mg} \mathrm{dL}^{-1}\right)$ & Glucose (mg dL ${ }^{-1}$ ) & Hematocrit (\%) \\
\hline \multirow[t]{4}{*}{50} & Control & $4.45 \pm 0.76 \mathrm{Aa}$ & $44.06 \pm 29.25 \mathrm{Ab}$ & $41.90 \pm 22.03 \mathrm{Bb}$ & $36.96 \pm 1.53 \mathrm{ABa}$ \\
\hline & AT & $7.67 \pm 5.26 \mathrm{Aa}$ & $80.98 \pm 16.44 \mathrm{Aa}$ & $104.59 \pm 58.17 \mathrm{Aa}$ & $27.08 \pm 3.17 \mathrm{Ab}$ \\
\hline & $24 \mathrm{~h} \mathrm{AT}$ & $4.95 \pm 3.28 \mathrm{Aa}$ & $34.59 \pm 12.34 \mathrm{Aa}$ & $45.48 \pm 6.28 \mathrm{Ab}$ & $26.22 \pm 1.17 \mathrm{Ab}$ \\
\hline & $96 \mathrm{~h} \mathrm{AT}$ & $5.33 \pm 3.23 \mathrm{Aa}$ & $104.58 \pm 20.35 \mathrm{Aa}$ & $40.82 \pm 3.97 \mathrm{Ab}$ & $22.17 \pm 2.50 \mathrm{ABb}$ \\
\hline \multirow[t]{4}{*}{80} & Control & $6.48 \pm 2.22 \mathrm{Aa}$ & $34.01 \pm 16.98 \mathrm{Ab}$ & $86.72 \pm 32.95 \mathrm{ABab}$ & $39.83 \pm 2.44 \mathrm{Aa}$ \\
\hline & AT & $7.53 \pm 7.04 \mathrm{Aa}$ & $98.76 \pm 43.48 \mathrm{Aa}$ & $142.94 \pm 18.65 \mathrm{Aa}$ & $27.17 \pm 4.02 \mathrm{Ab}$ \\
\hline & $24 \mathrm{~h} \mathrm{AT}$ & $4.42 \pm 3.56 \mathrm{Aa}$ & $50.62 \pm 33.70 \mathrm{Aa}$ & $45.83 \pm 16.28 \mathrm{Ab}$ & $23.00 \pm 3.30 \mathrm{Ab}$ \\
\hline & $96 \mathrm{~h} \mathrm{AT}$ & $5.53 \pm 3.45 \mathrm{Aa}$ & $77.45 \pm 27.71 \mathrm{Aa}$ & $50.49 \pm 32.09 \mathrm{Ab}$ & $25.75 \pm 2.77 \mathrm{Ab}$ \\
\hline \multirow[t]{4}{*}{110} & Control & $4.59 \pm 2.90 \mathrm{Aa}$ & $36.45 \pm 40.39 \mathrm{Ab}$ & $120.06 \pm 39.85 \mathrm{Aa}$ & $35.50 \pm 1.29 \mathrm{ABa}$ \\
\hline & AT & $4.78 \pm 3.58 \mathrm{Aa}$ & $75.62 \pm 23.46 \mathrm{Aa}$ & $105.37 \pm 68.17 \mathrm{Aa}$ & $27.00 \pm 0.82 \mathrm{Ab}$ \\
\hline & $24 \mathrm{~h} \mathrm{AT}$ & $2.47 \pm 2.20 \mathrm{Aa}$ & $82.61 \pm 30.04 \mathrm{Aa}$ & $46.12 \pm 9.68 \mathrm{Ab}$ & $22.00 \pm 4.08 \mathrm{Abc}$ \\
\hline & 96 h AT & $4.07 \pm 3.01 \mathrm{Aa}$ & $85.64 \pm 34.46 \mathrm{Aa}$ & $62.43 \pm 47.59 \mathrm{Ab}$ & $21.25 \pm 3.30 A B C$ \\
\hline \multirow[t]{4}{*}{140} & Control & $3.47 \pm 3.64 \mathrm{Aa}$ & $50.54 \pm 38.11 \mathrm{Ab}$ & $100.99 \pm 16.21 \mathrm{Aa}$ & $32.50 \pm 3.87 \mathrm{Ba}$ \\
\hline & AT & $9.96 \pm 12.07 \mathrm{Aa}$ & $71.70 \pm 22.66 \mathrm{Aa}$ & $78.15 \pm 34.17 \mathrm{Aab}$ & $27.75 \pm 1.26 \mathrm{Ab}$ \\
\hline & $24 \mathrm{~h} \mathrm{AT}$ & $4.56 \pm 3.21 \mathrm{Aa}$ & $93.94 \pm 37.64 \mathrm{Aa}$ & $48.87 \pm 4.96 \mathrm{Abc}$ & $25.50 \pm 3.79 \mathrm{Ab}$ \\
\hline & $96 \mathrm{~h} \mathrm{AT}$ & $4.53 \pm 3.72 \mathrm{Aa}$ & $66.30 \pm 16.40 \mathrm{Aa}$ & $44.77 \pm 7.45 \mathrm{Ac}$ & $19.50 \pm 1.00 \mathrm{BC}$ \\
\hline \multirow[t]{4}{*}{170} & Control & $2.33 \pm 1.00 \mathrm{AC}$ & $66.54 \pm 42.66 \mathrm{Ab}$ & $115.11 \pm 23.90 \mathrm{Aa}$ & $36.50 \pm 2.52 \mathrm{ABa}$ \\
\hline & AT & $44.18 \pm 7.03 \mathrm{Ba}$ & $133.31 \pm 13.90 \mathrm{Aa}$ & $132.13 \pm 14.12 \mathrm{Aa}$ & $24.50 \pm 2.65 \mathrm{Ab}$ \\
\hline & $24 \mathrm{~h} \mathrm{AT}$ & $4.10 \pm 3.36 \mathrm{Abc}$ & $105.59 \pm 17.75 \mathrm{Aa}$ & $83.90 \pm 39.06 \mathrm{Aab}$ & $22.75 \pm 2.63 \mathrm{Abc}$ \\
\hline & 96 h AT & $11.64 \pm 7.59 \mathrm{Ab}$ & $79.00 \pm 49.64 \mathrm{Aa}$ & $52.26 \pm 6.33 \mathrm{Ab}$ & $18.33 \pm 2.08 \mathrm{BC}$ \\
\hline \multicolumn{2}{|l|}{ Density (D) } & $p<0.05$ & $p<0.05$ & $p<0.01$ & $p<0.001$ \\
\hline \multicolumn{2}{|c|}{ Transportation period (P) } & $p<0.01$ & $p<0.001$ & $p<0.001$ & $p<0.001$ \\
\hline \multicolumn{2}{|c|}{ DxPInteraction } & $p<0.05$ & ns & $p<0.05$ & $p<0.01$ \\
\hline
\end{tabular}

Different uppercase superscript letters indicate significant differences between densities. Lowercase letters denote difference between transportation periods (Tukey, $\mathrm{p}<0.05$ ); ns (not significant).

\section{DISCUSSION}

The mortality of fingerlings at higher densities demonstrated that transportation at such densities had irreversible stress effects or osmoregulatory dysfunction on the fish, which Lim et al. (2003) stated as the most frequent cause of post-shipment fish mortality. In general, small fish are not affected by transportation stress and most mortality occurs during recovery, possibly due to additional stress from acclimation (Froese 1988), which results in immune suppression (Harmon 2009) and even death. Although no mortality was observed for juveniles, cortisol increased after transportation and had not returned to the initial values by the end of the recovery period. This finding indicates that the loading capacity in closed systems (plastic bags) came very close to the maximum at $170 \mathrm{~g} \mathrm{~L}^{-1}$. Our results are consistent with those reported by Lima and Oliveira (2018) in juvenile pirarucu $(9.0 \mathrm{~kg})$ transported at a density of $160 \mathrm{~g} \mathrm{~L}^{-1}$ in open systems with no mortality. This demonstrates that pirarucu can withstand higher stocking densities as they grow.

As expected, water quality deteriorated after transportation. Higher water temperature after transporting fingerlings or juveniles was due to the plastic bags being exposed to air temperature, which is frequently higher than the water temperature in the study area. The same trend has been observed for pirarucu transportation in an open system (Lima and Oliveira 2018).

A higher water oxygen concentration after transportation is commonly reported for fish transported in plastic bags as pure oxygen is injected into water (Carneiro et al. 2009; Zeppenfeld et al. 2014). Our oxygen concentrations after transporting both fingerlings and juveniles were at the levels recommended for fish (Boyd 1979), which indicates that the water oxygen was not a limiting factor for transportation (Harmon 2009). The higher oxygen levels after transportation in the juvenile bags as compared to fingerlings, can be related 
to the characteristic of this species to become obligate airbreathing as they grow $(1 \mathrm{~kg})$, whereas fingerling $(24.5 \mathrm{~g})$ respiration is aquatic (Brauner et al. 2004).

The decrease in water $\mathrm{pH}$ after transporting in both fingerlings and juveniles was similar to what was reported by Treasurer (2012) for the transportation of Gadus morhua Linnaeus 1758, and by Carneiro et al. (2009) for Rhamdia quelen (Quoy and Gaimard 1824). The drop in water $\mathrm{pH}$ is probably related to the increased $\mathrm{CO}_{2}$ levels observed at the end of transport, which result from animal metabolism (Harmon 2009). As the drop in $\mathrm{pH}$ was similar for all the tested densities, it is apparently not related to juvenile mortality. As pirarucu is a native fish species to the Amazon basin, where water is naturally acidic (Gomes et al. 2003), it tolerates $\mathrm{pH}$ values below 7.0.

The unionized ammonia levels increased at higher densities in both fingerlings and juveniles, even after the 24-hour fasting period recommended prior to fish transportation (Conte 2004; Harmon 2009), as also reported by Carneiro et al. (2009) with Rhamdia quelen. During transportation of the juveniles, the unionized ammonia levels were below $2.0 \mathrm{mg} \mathrm{L}^{-1}$, which was the maximum concentration reported by Cavero et al. (2004) and did not cause mortality in the $2.6 \mathrm{~kg}$ juvenile pirarucu (Cavero et al. 2004). Unionized ammonia concentration during transportation was $2.39 \mathrm{mg}$ $\mathrm{L}^{-1}$ for fingerlings at $95 \mathrm{~g} \mathrm{~L}^{-1}$ with no recorded mortality, which demonstrates that it was within the limits for pirarucu of $24 \mathrm{~g}$ at this density. At the densities for which mortality occurred, concentrations went above $3.0 \mathrm{mg} \mathrm{L}^{-1}$, a high value, but still below those reported by Lima and Oliveira (2018) for transportation water of juvenile pirarucu $(9 \mathrm{~kg})$, with no reported mortality. Nevertheless, younger individuals are frequently more sensitive to ammonia (Salin and Williot 1991), which indicates that unionized ammonia may be one of the factors that contributed to the mortality that we observed during the transportation of fingerlings.

An increase in $\mathrm{CO}_{2}$ during transportation was expected as a result of fish metabolism in a closed system. Although $\mathrm{CO}_{2}$ accumulation in water may be more critical for fish than ammonia (Wedemeyer 1996; Portz et al. 2006), it occurred uniformly at all fingerling densities. Although $\mathrm{CO}_{2}$ levels increased at higher juvenile densities, they generally did not exceed the values recommended by Wedemeyer (1996) as being safe for freshwater fish, i.e., below 20-30 $\mathrm{mg} \mathrm{L}^{-1}$, so that we suggest that it was not responsible for the observed fingerling mortalitiy. Additionally, a sharp rise in water $\mathrm{CO}_{2}$ levels may result in blood acidification and higher hematocrit values (Paterson et al. 2003), which was not observed in our juveniles. Yet the low hematocrit values after transportation and during the recovery period demonstrate that the $\mathrm{CO}_{2}$ values were at the limit tolerated by the species.
Although longer fasting periods are recommended for larger fish (Froese 1998), ammonia excretion was greater in the fingerling pirarucu than in the juveniles. Furthermore, fingerling oxygen consumption and $\mathrm{CO}_{2}$ production were higher than in the juveniles, even though they were handled similarly and at lower densities. This scenario suggests that younger pirarucu have higher metabolic rates which, in turn, result in faster water deterioration and, thus, less carrying capacity, as reported by Berka (1986) and Portz et al. (2006), who recommend lower densities for smaller individuals.

Physiological parameters are good indicators of the fish stress response and can be influenced by water quality deterioration, a critical factor in fish transportation (Sampaio and Freire 2016), as observed in all our treatments. Of all the measured parameters, lactate increased significantly after transportation for both fingerlings and juveniles, and did not returned to the initial values until the end of the recovery period, as also reported by Gomes et al. (2006) in pirarucu transportation at different salinities. Similar to that observed by Gomes et al. (2006), lactate was the strongest physiological response in pirarucu because it had not returned to the initial values after the recovery period. Higher post-transportation lactate levels indicate lactic acid accumulation due to increased physical activity (Barton et al. 2002; Dobsikova et al. 2009), which is possibly related to capture, handling and confinement stress during transportation (Harmon 2009; Iversen et al. 2005; Portz et al. 2006). This further stresses the need to use anaerobic metabolism when the aerobic metabolism cannot provide fish with enough energy (Oliveira et al. 2018). Additionally, increased unionized ammonia in the water may result from fish agitation (Portz et al. 2006), and, consequently, higher lactate levels after transportation. A change in lactate levels is a frequent stress response in fish (Ross and Ross 2008) and may be quite variable. Atlantic salmon (Iversen et al. 2005) and Paralichthys olivaceus (Temminck and Schlegel, 1846) (Hur et al. 2006) displayed similar behavior after transportation, whereas Brandão et al. (2006) and Lima and Oliveira (2018) reported lower lactate levels after the transportation of larger pirarucu. Finally, fish in stressful situations may use lactate to produce glucose (Mommsem et al. 1999), which is an important energy source in the metabolic processes of pirarucu that face stress (Gomes et al. 2006).

As observed in most of our treatments, rising levels of glucose, but not of cortisol, were reported for pirarucu (34 g) by Brandão et al. (2006), who attributed this increase to catecholamines. Catecholamines are responsible for the immediate increase in blood glucose levels, whereas cortisol maintains high levels for longer (Barton et al. 2002), which may explain the return of the blood glucose levels to the initial values during the recovery period. Catecholamines release is also responsible for higher blood lactate levels (Ross and Ross 2008), which may explain the increase in lactate we observed. In general, a rise in blood glucose levels is attributed to fish 
needing to maintain the energy levels required for metabolic activities and, with a startle response, to provide the sudden high demand from muscles (Brandão et al. 2006). Gomes et al. (2003) have attributed high glucose levels in pirarucu $(1.1 \mathrm{~kg})$, even during the recovery period, to continuous stress from handling to perform blood sampling. Conversely, Lima and Oliveira (2018) did not observe any alterations in blood glucose during pirarucu transportation. Generally no pattern appears in the variation of blood glucose levels as a fish response to stressors, not even in the same experimental group (Mommsem et al. 1999; Ross and Ross 2008). The different responses of glucose levels to transportation stress in pirarucu may be related to both nutritional status and developmental stage, and should be analyzed together with other physiological parameters (Mommsem et al. 1999; Barton et al. 2002).

As observed in our juvenile treatments, Carneiro et al. (2009) found that the cortisol level peaked at the highest density of Rhamdia quelen, but with no mortality. Nevertheless, higher cortisol levels in our juveniles at $170 \mathrm{~g} \mathrm{~L}^{-1}$ and changes in water quality parameters, especially unionized ammonia, indicate that the system carrying capacity came close to its maximum. As in our juvenile densities up to 140 $\mathrm{g} \mathrm{L}^{-1}$, Gomes et al. (2003) did not report any cortisol level alteration in pirarucu $(1.1 \mathrm{~kg})$ transported in plastic bags either with or without oxygen addition. Hence pirarucu may be capable of maintaining low cortisol levels when they are exposed to stressors for short times (Gomes et al. 2003; Lima and Oliveira 2018).

Brandão et al. (2006) and Lima and Oliveira (2018) reported increased hematocrit in pirarucu after transportation, and related this hemoconcentration to increased oxygen demand to recover from exposure to a stressor. However we transported pirarucu in plastic bags with supersaturated oxygen and, as changes in cortisol levels were minor, fish may not have activated such a route to increase oxygen availability in blood. Furthermore, pirarucu is a freshwater species and, as transportation took place without adding salt to water, water uptake by fish took place to maintain osmoregulation (Harmon 2009; Silveira et al. 2009). This caused hemodilution, which could explain the drop in hematocrit in our study.

\section{CONCLUSIONS}

As pirarucu had increased blood glucose and lactate levels in all our treatments, but without high cortisol levels in most treatments, a secondary stress response was observed after transportation. However, the blood glucose levels returned to the initial values by $96 \mathrm{~h}$, and the lower cortisol and lactate levels demonstrated how fast pirarucu can recover from a stressful situation. Densities up to $95 \mathrm{~g} \mathrm{~L}^{-1}$ can be used safely to transport fingerling pirarucu. The $110 \mathrm{~g} \mathrm{~L}^{-1}$ density presented low mortality, but cortisol levels did not increase, and physiological parameters did not differ from fish at lower densities, save ammonia levels, and should be used with caution, preferably with longer fasting periods. The $140 \mathrm{~g} \mathrm{~L}^{-1}$ density is not recommended for transporting fingerling pirarucu, but densities up to $140 \mathrm{~g} \mathrm{~L}^{-1}$ may be safely used to transport juveniles. The $170 \mathrm{~g} \mathrm{~L}^{-1}$ density presented no mortality for juveniles, but increased the cortisol which remained higehr than initial values by the end of the recovery period. These findings indicate that the system carrying capacity at this density came close to its maximum and is, thus, not recommended for transporting juvenile pirarucu. Finally, as the lactate and hematocrit values had not returned to the initial values after the recovery period, we recommend avoiding fish being exposed to other stressors during this period.

\section{ACKNOWLEDGMENTS}

This study was funded by Ministério da Agricultura, Pecuária e Abastecimento, through Conselho Nacional de Desenvolvimento Científico e Tecnológico (CNPq) (grant no. 457465/2012-3), and by Serviço Brasileiro de Apoio às Micro e Pequenas Empresas (SEBRAE), through Fundação de Apoio Científico e Tecnológico do Tocantins (FAPTO) (agreement 9/2012, grant no. 2538/2012).

\section{REFERENCES}

Barton, B.A.; Morgan, J.D.; Vijayan, M.M. 2002. Physiological and Condition-Related Indicators of Environmental Stress in Fish. In: Adams, S. M. (Ed.) Biological Indicators of Aquatic Ecosystem Health. American Fisheries Society, Bethesda, p.111-148.

Berka, R. 1986. The Transport of Live Fish. A Review. EIFAC Technical Paper/FAO, Rome, 79p.

Boyd, C.E.; Lichtkoppler, F. 1979. Water quality management in pond fish culture. Research and Development Series 22. International Center for Aquaculture, Agricultural Experimental Station, University of Auburn, Alabama, 32p.

Brandão, F.R.; Gomes, L.D.C.; Chagas, E.C. 2006. Respostas de estresse em pirarucu (Arapaima gigas) durante práticas de rotina em piscicultura. Acta Amazonica 36: 349-356.

Brauner, C.J.; Matey, V.; Wilson, J.M.; Bernier, N.J.; Val, A.L. 2004. Transition in organ function during the evolution of airbreathing: insights from Arapaima gigas, an obligate air-breathing teleost from the Amazon. Journal of Experimental Biology, 207: 1433-1438.

Carneiro, P.C.F.; Kaiseler, P.H.S.; Swarofsky, E.A.C.; Baldisserotto, B. 2009. Transport of jundiá Rhamdia quelen juveniles at different loading densities: water quality and blood parameters. Neotropical Ichthyology, 7: 283-288.

Cavero, B.A.S.; Pereira-Filho, M.; Bordinhon, A.M.; Fonseca, F.A.L.; Ituassú, D.R.; Roubach, R.; Ono, E.A. 2004. Tolerância de juvenis de pirarucu ao aumento da concentração de amônia em ambiente confinado. Pesquisa Agropecuaria Brasileira, 39: 513-516. 
Conte, F.S. 2004. Stress and the welfare of cultured fish. Applied Animal Behaviour Science, 86: 205-223.

Cupp, A.R.; Fredricks, K.T.; Porcher, S.T.; Smerud, J. R.; Hartleb, C.F.; Gaikowski, M.P. 2017. Survival and behavioural responses of cool and warm water fish sedated with AQUI-S 20E (10\% Eugenol) at high loading densities. Aquaculture Research, 48: 1576-1589.

Dobšíková, R.; Svobodová, Z.; Bláhová, J.; Modrá, H.; Velíšek, J. 2009. The effect of transport on biochemical and haematological indices of common carp (Cyprinus carpio L.). Czech Journal of Animal Science, 11: 510-518.

Froese, R. 1988. Relationship between body weight and loading densities in fish transport using the plastic bag method. Aquaculture Research, 19: 275-281.

Gomes, L.C.; Roubach, R.; Cavero, B.A.S.; Pereira-Filho, M.; Urbinati, E.C. 2003. Transport of pirarucu Arapaima gigas juveniles in plastic bags. Acta Amazonica, 33: 637-642.

Gomes, L.C.; Chagas, E.C.; Brinn, R.P.; Roubach, R.; Coppati, C.E.; Baldisserotto, B. 2006. Use of salt during transportation of air breathing pirarucu juveniles (Arapaima gigas) in plastic bags. Aquaculture, 256: 521-528.

Harmon, T.S. 2009. Methods for reducing stressors and maintaining water quality associated with live fish transport in tanks: a review of the basics. Reviews in Aquaculture, 1: 58-66.

Hur, J.W.; Park, I.; Chang, Y.J. 2006. Physiological responses of the olive flounder, Paralichthys olivaceus, to a series stress during the transportation process. Ichthyological Research, 54: 32-37.

IBGE. 2018. Instituto Brasileiro de Geografia e Estatística. Produção da pecuária municipal (https://sidra.ibge.gov.br/Tabela/3940). Acessed on 05 May 2020.

Iversen, M.; Finstad, B.; Mckinley, R.S.; Eliassen, R.A.; Tuff, K.; Evjen, T. 2005. Stress responses in Atlantic salmon (Salmo Salar L.) smolts during commercial well boat transports, and effects on survival after transfer to sea. Aquaculture, 243: 373-382.

King, H.R. 2009. Fish transport in the aquaculture sector: an overview of the road transport of Atlantic salmon in Tasmania. Journal of Veterinary Behavior: Clinical Applications and Research, 4: 163-168.

Lim, L.C.; Dhert, P.; Sorgeloos, P. 2003. Recent developments and improvements in nornamental fish packaging systems for air transport. Aquaculture Research, 34: 923-935.

Lima, A.F.; Oliveira, H.J.B. 2018. Effect of density on survival, physiological parameters and water quality during pirarucu transportation in open system. Aquaculture Research, 49: 947-952.

Mommsen, T.P.; Vijayan, M.M.; Moon, T.W. 1999. Cortisol in teleosts: dynamics, mechanisms of action, and metabolic regulation. Reviews in Fish Biology and Fisheries, 9: 211-268.
Oliveira, B.L.; Souza, R.M.; Silva Filho, J.P.; Gomes, L.C.; Fernandes, L.F.L. 2018. Hematological and biochemical characteristics during the transport of dog snapper Lutjanus jocu (Perciformes: Lutjanidae). Neotropical Ichthyology, 16: 1-8.

Paterson, B.D.; Rimmer, M.A.; Meikle, G.M.; Semmens, G.L. 2003. Physiological responses of the Asian sea bass, Lates calcarifer, to water quality deterioration during simulated live transport: acidosis, red-cell swelling, and levels of ions and ammonia in the plasma. Aquaculture, 218: 717-728.

Portz, D.E.; Woodley, C.M.; Cech Jr., J.J. 2006. Stress-associated impacts of short-term holding on fishes. Reviews in Fish Biology and Fisheries, 16: 125-170.

R Core Team. 2016. A Language and environment for statistical computing. R Foundation for Statistical Computing, Vienna, Austria.

Rebelatto Junior, I.A.; Lima, A.F.; Rodrigues, A.P.O.; Maciel, P.O.; Kato, H.C.A.; Mataveli, M.; et al. 2015. Reprodução e Engorda do Pirarucu: Levantamento de Processos Produtivos e Tecnologias. 1st ed. Embrapa, Brasília, 102p.

Ross, L.G.; Ross, B. 2008. Anaesthetic and Sedative Techniques for Aquatic Animals. 3rd ed. Blackwell Publishing, Oxford, 120p.

Salin, D.; Williot, P. 1991. Acute toxicity of ammonia to Siberian sturgeon Acipenser baeri. In: Williot, P. (Ed.). Acipenser. Cemagref Publication, France, p.153-167.

Sampaio, F.D.F.; Freire, C.A. 2016. An overview of stress physiology of fish transport: changes in water quality as a function of transport duration. Fish and Fisheries, 17: 1055-1072.

Silveira, U.S.; Logato, P.V.R.; Pontes, E.D.C. 2009. Fatores estressantes em peixes. Revista Eletrônica Nutritime, 6: 10011017.

Treasurer, J.W. 2012. Changes in ph during transport of juvenile cod Gadus morhua L. and stabilisation using buffering agents. Aquaculture, 330-333: 92-99.

Wedemeyer, G.A. 1996. Physiology of fish in intensive culture systems. Chapman \& Hall, New York, 232p.

Zeppenfeld, C.C.; Toni, C.; Becker, A.G.; Miron, D.S.; Parodi, T.V.; Heinzmann, B.M.; et al. 2014. Physiological and biochemical responses of silver cat fish, Rhamdia quelen, after transport in water with essential oil of Aloysia triphylla. Aquaculture, 418-419: 101-107.

RECEIVED: $28 / 01 / 2020$

ACCEPTED: 16/07/2020

ASSOCIATE EDITOR: Rodrigo R. do Valle 